\title{
¿Hacia la academización de las facultades de derecho en Chile? Un análisis teórico y comparado del conflicto de las profesiones
}

\author{
Fernando Muñoz León*
}

\begin{abstract}
RESUMEN
Las facultades de derecho chilenas están viviendo un proceso de cambio consistente en la proliferación al interior de ellas de académicos de dedicación completa y exclusiva. Ello involucra una cierta pérdida de protagonismo dentro de las mismas de los docentes que dividen su tiempo entre los tribunales y las aulas universitarias. ¿Estamos ante la profesionalización de la docencia jurídica? Este ensayo sugiere entender este fenómeno más bien como un caso de conflicto entre dos profesiones, los "académicos" y los "docentes litigantes", al interior de las facultades. Para comprender el posible destino de este "conflicto de las profesiones", este ensayo propone poner atención a las prácticas académicas de Estados Unidos, cuyas facultades de derecho atravesaron este proceso de academización hacia fines del siglo XIX y principios del siglo XX.
\end{abstract}

Academia jurídica - profesiones - sociología jurídica

\section{Towards the academization of law faculties in Chile? A theoretical and comparative analysis of the conflict of the professions}

\begin{abstract}
Chilean law faculties are undergoing a process of change that consists in the proliferation within them of full-time academics. This involves a certain loss of importance within them of instructors that split their time between courts and classrooms. Are we witnessing the professionalization of legal instruction? This essay suggests understanding this phenomenon rather as a case of conflict between two professions, the 'academics' and the 'litigant teachers', within the faculties. To understand the possible outcome of this 'conflict of the professions', this essay proposes to pay attention to the academic practices of the United States, whose law faculties went through this process of academization at the end of the $19^{\text {th }}$ and the beginning of the $20^{\text {th }}$ centuries.
\end{abstract}

Legal academia - professions - legal sociology

* Profesor Auxiliar, Facultad de Ciencias Jurídicas y Sociales, Universidad Austral de Chile. Doctor en Derecho, Yale Law School. Correo electrónico: fernando.munoz@uach.cl.

Artículo recibido el 30 de agosto de 2013 y aceptado para su publicación el 28 de mayo de 2014. 


\section{INTRODUCCIÓN}

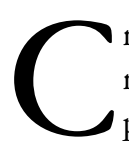

reo posible sostener que la academia jurídica en Chile está experimentando un rápido proceso de cambio. Este podría ser descrito, preliminarmente, como un proceso de profesionalización, entendiendo por tal un proceso de especialización del trabajo; en este caso, del trabajo académico. Así, y en un grupo importante (pero en ningún caso universal) de facultades de derecho, de ser labores realizadas en los intersticios de la actividad profesional del abogado litigante ${ }^{1}$, las siguientes están comenzando a ser labores realizadas a tiempo completo por académicos: la producción de conocimiento jurídico en la forma de publicaciones especializadas; la transmisión de dicho conocimiento a los futuros profesionales en la forma de docencia universitaria, y al resto de la comunidad mediante la extensión; y la gestión misma de las instituciones de educación superior, incluyendo la planificación curricular y metodológica de la docencia antes dicha. Las facultades de derecho se estarían sumando a aquella visión convencional según la cual "sin un profesorado sólido, bien preparado y comprometido, ninguna institución académica o sistema de educación superior puede ser exitoso” 2 .

A manera de hipótesis respecto de las causas de dichos cambios, me permitiría sugerir que en ellos confluyen diversos fenómenos relacionados con la consolidación institucional del sistema universitario chileno. Entre estos fenómenos se encontrarían la masificación de las escuelas de derecho, que a su vez lleva a una competencia de mercado entre las mismas; la visibilización de dicha competencia mediante el surgimiento de variados rankings; la especialización de las tareas investigativas por exigencias de formación doctoral; la exclusividad contractual como estrategia monopólica de las facultades en la competencia por los mejores docentes e investigadores; el recurso a la acreditación como mecanismo de certificación de la calidad de la enseñanza; las demandas características de los procesos de acreditación; y -last but not least-el trasplante de objetivos, prácticas y expectativas de carácter académico provenientes en forma directa de otras latitudes e indirectamente de otras disciplinas. Mientras que algunas de estas dinámicas -en particular, las primeras- no apuntan con necesidad a la proliferación en las facultades de derecho de académicos de dedicación completa y exclusiva, las últimas sí parecieran hacerlo en el presente, imprimiéndole una orientación más clara al conjunto de dichos procesos.

${ }^{1}$ Emplearé aquí esta expresión para denominar al heterogéneo conjunto de quienes, según la expresión tan habitual, "ejercen libremente la profesión de abogado". Por supuesto, no creo que exista una profesión de abogado. Enseñar derecho también es ejercer la profesión. Lo es, también, desempeñarse como juez, notario, defensor público, fiscal, o abogado corporativo. Ahora bien, para efectos de esta discusión, subsumiré a esta abigarrada variedad de formas de ejercer la profesión dentro de la figura del abogado litigante. Escojo a dicha figura porque a mi juicio es la manifestación alternativa al académico más frecuente en las facultades. Asimismo, escojo a una sola figura, que estimo suficientemente representativa del resto, para simplificar lingüísticamente mi discusión del conflicto entre académicos y no académicos.

${ }^{2}$ Altbach, P. et al. (eds), Paying the Professoriate: A Global Comparison of Compensation and Contracts, New York, Routledge, 2012, p. 3. 
Ahora bien, formulada por un académico, ¿no sería la observación con que comienza este artículo un ejemplo de historia whig, un caso de autocomplaciente descripción de la realidad como un constante progreso hacia el presente? A fin de evitar caer en el whiggismo, voy a sugerir una forma alternativa de entender el proceso de cambio en cuestión. Esta perspectiva involucra reconocer que no corresponde calificar al litigante que hace docencia como un no profesional. A la luz de esta premisa, es más adecuado señalar que el proceso recién reseñado consiste, en estricto rigor, no tanto en una profesionalización a secas, sino más bien en un conflicto. No un conflicto de las facultades ${ }^{3}$, lógicamente, sino un conflicto dentro de ellas entre dos grupos de profesionales del derecho: ¿los "docentes litigantes", por un lado, y los "académicos", por el otro? Ellos no necesariamente entran en conflicto en un sentido subjetivo, es decir, en el sentido de que sus voluntades y sus ánimos entren en mutuo combate ${ }^{4}$, sino que entran en conflicto en un sentido objetivo: los unos desplazan a los otros de posiciones de influencia y poder anteriormente sostenidos sin mayores cuestionamientos. Para comprender esta afirmación, acometeré un análisis teórico del concepto de profesionalización.

Además de observar este fenómeno, en este trabajo quiero invitar a la reflexión sobre algunas preguntas prospectivas. ¿Qué implicancias tiene para nuestro sistema universitario el paulatino desplazamiento de los "litigantes" a manos de los "académicos”? ¿En qué podría consistir el nuevo modelo de Facultad de Ciencias Jurídicas nacional? ¿Qué estructuras internas y externas adoptará para llevar a cabo las funciones de producción académica, entrenamiento profesional, y vinculación con el entorno social que normalmente se esperan de las instituciones de educación superior? ¿Qué oportunidades y peligros nos aguardan con este proceso? Intentaré sugerir respuestas a dichas preguntas a partir de un análisis comparado de la academia jurídica norteamericana. He escogido dicho caso debido a que, como he observado en un anterior artículo, la academia norteamericana ya atravesó un proceso de academización hacia fines del siglo XIX y principios del siglo XX; proceso que estuvo guiado por los argumentos y discursos justificatorios ofrecidos por Christopher Columbus Langdell y Oliver Wendell Holmes ${ }^{5}$. Aquí dedicaré mi atención, en consecuencia, no al proceso de academización sino al resultado del mismo. Por cierto, al fijar mi atención en la estructura y prácticas de las facultades de derecho se hace innecesario que analice las diferencias y similitudes entre nuestro sistema jurídico y el norteamericano ${ }^{6}$.

${ }^{3}$ La referencia, por supuesto, es a Kant, I., El conflicto de las facultades, Madrid, Alianza Editorial, 2003.

${ }^{4}$ Aunque tampoco es imposible que ello ocurra. En todo caso, el estudio casuístico de conflictos subjetivos entre "docentes litigantes" y "académicos" no forma parte de los objetivos de este trabajo.

5 Muñoz, F., "Langdell's and Holmes's influence on the institutional and discursive conditions of American legal scholarship”, Revista Chilena de Derecho, Vol. 38, 2011, pp. 217-237.

${ }^{6}$ Estas diferencias y similitudes son habitualmente administradas mediante la dicotomía entre derecho continental y common law. El análisis clásico de dicha dicotomía se encuentra en Zweigert, K., y Kötz, H., Introduction to Comparative Law, Oxford, Oxford University Press, 1998. Se puede encontrar una evaluación contemporánea de la misma en Pargendler, M., "The Rise and Decline of Legal Families”, American Journal of Comparative Law, Vol. 60, 2012, pp. 1043-1074. 


\section{EL SISTEMA DE LAS PROFESIONES Y LA COMPETENCIA POR JURISDICCIÓN}

El estudio de las profesiones ha sido desde temprano una de las preocupaciones de la sociología. Los propios orígenes de esta disciplina están vinculados a los esfuerzos por comprender las consecuencias de la división del trabajo sobre la estructura social. Es lógico que esta preocupación haya llevado a los sociólogos a estudiar una de las manifestaciones de dicha división, la configuración de diversas disciplinas o profesiones. Max Weber, Emile Durkheim y Georg Simmel contribuyeron así a construir los cimientos de la literatura sobre las profesiones ${ }^{7}$.

La literatura contemporánea sobre profesiones, por supuesto, ha recorrido un largo camino desde entonces. Podemos encontrar una síntesis crítica del estudio de las profesiones en la propuesta de Andrew Abbott. Según este autor, en Estados Unidos la literatura sobre las profesiones y los procesos de profesionalización ha transitado por las siguientes perspectivas: funcionalista, estructuralista, monopolista, y culturalista. Para la perspectiva funcionalista, una profesión es un mecanismo para controlar la relación asimétrica entre expertos y clientes, y la profesionalización es simplemente la evolución de las garantías estructurales de ese control. Para la perspectiva estructuralista, la profesión es una forma de control ocupacional; la comprensión del proceso de profesionalización involucra la explicación de los pasos que atraviesa una actividad en el desarrollo de mecanismos de control ${ }^{8}$. Para la perspectiva monopolista, el desarrollo de dichos mecanismos no es natural o espontáneo, sino que el fruto de un deliberado propósito de dominio o autoridad; las profesiones son grupos corporativos que buscan controlar rentas y estatus mediante un dominio ocupacional. Finalmente, observa Abbott, autores que trabajan en la perspectiva culturalista han retornado al foco en el rol de la experticia como un determinante de las relaciones sociales, enfatizando la autoridad cultural de las profesiones?.

Abbott critica a las perspectivas antes identificadas, particularmente a la estructuralista, por su excesivo foco en profesiones construidas desde la autonomía social, dejando de lado a profesiones construidas al alero de la institucionalidad estatal, típicas de Europa continental. En términos más generales, critica a todas ellas por analizar y teorizar a las profesiones de manera individual, atomizada. En contraste con ello, Abbott propone entender lo profesional como un campo en que existen diversas relaciones entre profesiones; más específicamente, como un ecosistema en el cual cada profesión

${ }^{7}$ Weber, M., El político y el científico, Madrid, Alianza Editorial, 2005; Weber, M., Economía y sociedad. Esbozo de sociología comprensiva, México, Fondo de Cultura Económica, 1964; Durkheim, É., La división social del trabajo, Madrid, Akal, 1987; Simmel, G., Sobre la individualidad y las formas sociales, Buenos Aires, Universidad Nacional de Quilmes, 2002.

${ }^{8}$ Esta tesis incluye la detección de varios hitos por los cuales en Estados Unidos se ha construido la identidad colectiva de las profesiones: apertura de escuelas de entrenamiento profesional, desplazamiento de las mismas hacia las universidades, asociaciones profesionales locales y nacionales, aprobación de leyes que requieren la obtención de un título para el ejercicio profesional, la aparición de códigos de ética profesional.

${ }^{9}$ Abbott, A., The System of Professions. An Essay on the Division of Expert Labor, Chicago, The University of Chicago Press, 1988, p. 15. 
compite con otras por un determinado coto laboral. Por esto, su foco es en el sistema de las profesiones, esto es en las relaciones de competencia, colaboración y jerarquía que se dan entre ellas; y el sitio donde explorar dichas relaciones es en el control por parte de una profesión de determinadas actividades, labores, o problemáticas, vínculo de control al que denomina jurisdicción. "Analizar el desarrollo de las profesiones es analizar cómo este vínculo es creado en el contexto laboral, cómo es anclado por la estructura social formal e informal, y cómo la interacción entre los vínculos jurisdiccionales de distintas profesiones determina la historia de las profesiones individuales en sî" ${ }^{10}$. En resumidas cuentas, la competencia por jurisdicción explica el sistema de las profesiones existente en un determinado contexto.

La propuesta teórica de Abbott nos sirve para conceptualizar de manera más certera el proceso de cambio identificado al principio de este trabajo. Lo que ocurre hoy en las facultades de derecho chilenas es un proceso de competencia entre dos profesiones, los litigantes docentes y los académicos, por reclamar eficazmente jurisdicción sobre las labores de docencia, investigación, y gestión universitaria. En efecto, la integración predominante del cuerpo docente por académicos de dedicación exclusiva conlleva una creciente exclusión de la conducción de la institución universitaria y de la enseñanza de los principales cursos de quienes litigan en tribunales o desempeñan otras funciones en el sistema judicial.

Quisiera examinar dos registros discursivos en los cuales esta competencia por jurisdicción al interior de las facultades de derecho ha sido visibilizada. En primer lugar, examinaré una discusión en un diario nacional entre decanos de facultades de derecho. A continuación, revisaré algunas actas de procesos de acreditación de facultades de derecho. Me parece que estos registros evidencian los imaginarios que, en representación de las dos profesiones en cuestión, se disputan el dominio jurisdiccional de las facultades de derecho.

El primer registro ofrece un enfrentamiento entre dos narrativas altamente idealizadas, casi míticas, que sustentan sus respectivas tesis sobre la orientación que debe tener la facultad de derecho en posturas sobre el personal que debe primar en ellas. Una de ellas, la que cabría calificar como tradicional, plantea que para que la facultad de derecho pueda estar exitosamente orientada al ejercicio profesional, ya sea litigioso o corporativo, ella debe estar construida en torno a la figura del litigante prestigioso devenido en educador en su tiempo libre. Esta narrativa ha sido formulada por Pablo Rodríguez, Decano de la Facultad de Derecho de la Universidad del Desarrollo, en diversas columnas editoriales en el diario El Mercurio. Allí ha afirmando que, en la facultad de derecho,

${ }^{10}$ Ídem, p. 20. Véase también ídem, p. 33: "Cada profesión está amarrada a un conjunto de labores por vínculos jurisdiccionales, cuyas fortalezas y debilidades están establecidas en el proceso mismo del trabajo profesional. Ya que ninguno de estos vínculos es absoluto o permanente, las profesiones en su conjunto componen un sistema interactivo, un ecosistema. Las profesiones compiten entre sí dentro de este ecosistema, y el éxito de una profesión refleja tanto la situación de sus competidores y la estructura del sistema mismo como los esfuerzos de la propia profesión. Cada cierto tiempo, tareas son creadas, abolidas o reformadas por fuerzas externas, con sus consiguientes desplazamientos y reajustes dentro del sistema de las profesiones”. 
"el investigador, el docente, el filósofo del derecho y el jurista son una excepción que enaltece la profesión", pero que "lo que Chile necesita" son "abogados para organizar y reglamentar con eficiencia la vida social"11. Asimismo, ha sostenido que los "académicos profesionales", "sin duda útiles en ciertas cátedras, nada o muy poco aportan a la hora de ejercer el derecho vivo, cuando la norma se confronta con la realidad" 12 .

La segunda narrativa, que podríamos calificar como revisionista, sostiene que solo mediante la incorporación de la figura transnacional del académico de tiempo completo, validada por las universidades más prestigiosas del mundo, la facultad de derecho contemporánea podrá alcanzar sus propósitos de una manera autorreflexiva o crítica. En el caso específico que me ocupa, esta narrativa fue planteada como una respuesta a Rodríguez por parte de Rodrigo Correa, Decano de la Facultad de Derecho de la Universidad Adolfo Ibáñez. Correa argumentó que "un sistema jurídico sano requiere de personas dedicadas a examinarlo críticamente", las cuales "deben contar con tiempo para el constante estudio, tanto del derecho nacional e internacional como de disciplinas importantes para el derecho", y deben tener "la libertad para criticar las decisiones que toman diversos actores del sistema jurídico"13. Como coronación de su argumento, Correa invocó explícitamente algunos modelos ultramarinos de docencia jurídica (Harvard, Oxford, Leiden, Alexander von Humboldt, París II, Pompeu Fabra), afirmando que "no debe extrañar que todas las facultades de derecho que gozan de prestigio más allá de sus fronteras" estén "integradas principalmente por académicos" 14 .

El segundo registro al que he hecho referencia está conformado por acuerdos de acreditación tomados por agencias acreditadoras privadas y, al menos en un par de casos, por la propia Comisión Nacional de Acreditación. Estos acuerdos, como es lógico, solo reflejan el caso de las facultades que han resuelto someterse al procedimiento de acreditación ${ }^{15}$, el que en nuestra actual legislación es voluntario ${ }^{16}$. Ellos evidencian la dispar evaluación que, en la actualidad, reciben las facultades, por parte de la institucionalidad regulatoria universitaria, de acuerdo con el número de su personal académico. Esto se comprueba, por ejemplo, observando la retórica que acompaña la evaluación de las carreras de pregrado que han obtenido el mayor número de años de acreditación.

${ }^{11}$ Rodríguez, P., "Misión profesional de la universidad”, El Mercurio, 23 de mayo de 2009, A 2.

12 Rodríguez, P., "Sistema jurídico chileno”, El Mercurio, 19 de enero de 2011, A 2.

${ }^{13}$ Correa, R., "Sistema jurídico chileno", El Mercurio, 20 de Enero de 2011, A 2.

${ }^{14}$ Ídem.

${ }^{15}$ De acuerdo con lo informado por la Comisión Nacional de Acreditación se han sometido a este proceso trece universidades, de entre las cuales en este momento dos gozan de una acreditación de dos años (Universidad de Antofagasta y Universidad Bernardo O'Higgins), una de tres años (Universidad de Antofagasta), una de cuatro años (Universidad Católica de la Santísima Concepción), dos de cinco años (Universidad Católica del Norte y Universidad Alberto Hurtado), cinco de seis años (Pontificia Universidad Católica de Valparaíso, Universidad Austral de Chile, Universidad de Concepción, Universidad de Los Andes, Universidad de Valparaíso), y una de siete (Universidad Diego Portales).

${ }^{16}$ Para una crítica de la voluntariedad en los procesos de acreditación, véase Muñoz, F., "La necesidad de regular la docencia universitaria en Chile: una propuesta de lege ferenda", Revista Chilena de Derecho, Vol. 39, 2012, pp. 891-907. 
Así, en un caso se señala que "la carrera cuenta con un cuerpo académico suficiente en cantidad y altamente calificado para sustentar el programa" y los "docentes cuentan con un elevado nivel y diversidad de formación disciplinaria, y con reconocida productividad en investigación" ${ }^{17}$. En otro, se afirma que el "cuerpo académico de la carrera es suficiente en cantidad, calidad y dedicación para implementar el plan de estudios” y que la "carga de trabajo está adecuadamente equilibrada entre las funciones de docencia, investigación y gestión que deben desempeñar" ${ }^{18}$. En un tercer ejemplo se observa que el “cuerpo académico es calificado y con un número creciente de postgraduados", así como que existe "un nivel de preparación muy bueno en un grupo importante de profesores con entusiasmo y vocación por lo que hacen"19. En otro caso se valora que la "dotación, dedicación y calificación del cuerpo docente es adecuada para llevar a cabo el plan de estudios que se ha propuesto la unidad", al tiempo que se lamenta "la disminución de profesores de jornada completa entre 2009 y $2010 " 20$. Respecto de otra institución se destaca que "el personal docente es calificado y competente, advirtiéndose el esfuerzo que se ha hecho para aumentar la dotación de profesores con postgrados en jornada completa" ${ }^{21}$. En un último ejemplo, el organismo acreditador valoró que la unidad académica en cuestión "cuenta con un número apropiado y calificado de profesores que le permite dar cumplimiento a las funciones de docencia”, destacando que ella tiene "una dotación de 19 jornadas completas, 13 medias jornadas y 2 con un cuarto de jornada, todos con estudios de postgrado", al tiempo que de "las jornadas completas y medias jornadas, la mayoría se encuentra adscrito a la carrera académica regular y realizan docencia e investigación" 22 .

¿Hay valoración por parte de los reguladores universitarios de la presencia de litigantes docentes en las facultades con alta acreditación? Al menos hay un caso en el que los acuerdos de acreditación destacan el que, junto a los profesores académicos, "existe un alto número de profesores vinculados con el ejercicio activo de la profesión, lo que es valorado por los estudiantes" 23 .

Viceversa, en el caso de una de las carreras que obtuvieron menor tiempo de acreditación se critica que "la cantidad de profesores y su dedicación no es suficiente para cumplir con la misión y objetivos en las distintas áreas del quehacer de la unidad”, observación que se respalda señalando que la unidad académica "tiene dos académicos de jornada completa (Decano y Jefa de Carrera) y diez profesores de media jornada"24,

\footnotetext{
${ }^{17}$ Qualitas, Acuerdo de Acreditación No 155: Pontificia Universidad Católica de Valparaíso, p. 7.

${ }^{18}$ Qualitas, Acuerdo de Acreditación $N^{\circ}$ 122: Universidad Austral de Chile, p. 5

${ }^{19}$ Comisión Nacional de Acreditación, Acuerdo de Acreditación $N^{\circ}$ 311: Universidad de Concepción, p. 4.

${ }^{20}$ Qualitas, Acuerdo de Acreditación $N^{\circ} 125$ : Universidad de los Andes, p. 4.

${ }^{21}$ Agencia Acreditadora de Chile, Acuerdo de Acreditación $N^{\circ}$ 101: Universidad de Valparaíso, p. 7

22 Agencia Acreditadora de Chile, Acuerdo de Acreditación $N^{\circ}$ 61: Universidad Diego Portales, p. 6.

${ }^{23}$ Qualitas, Acuerdo de Acreditación No 155: Pontificia Universidad Católica de Valparaíso, p. 7.

${ }^{24}$ Qualitas, Acuerdo de Acreditación No 161: Universidad Bernardo O'Higgins, 7.
} 
mientras que en el caso de otra carrera se criticó que "existen pocos académicos contratados en calidad de planta" y que "una baja cantidad tiene formación doctoral" 25 .

El debate entre decanos y las actas de acreditación sugieren que el triunfo en la competencia por jurisdicción entre las profesiones jurídicas al interior de las facultades de derecho estará del lado de aquella profesión que logre persuadir eficazmente a los diversos actores del sistema universitario - demandantes de educación, demandantes de literatura especializada, autoridades ministeriales, agencias reguladoras y fiscalizadoras, prensa especializada- de sus ventajas comparativas en la realización de las labores que componen el respectivo campo operativo; particularmente la formación de futuros profesionales jurídicos. ¿Quién está mejor capacitado para enseñar a nuestros futuros abogados, los litigantes docentes o los académicos? Esa es la pregunta que guía y seguirá guiando la competencia por jurisdicción al interior de las facultades.

\section{LA ACADEMIZACIÓN DE LAS FACULTADES EN ESTADOS UNIDOS Y SUS CRÍTICOS}

Hasta ahora hemos contemplado cómo nuestras facultades de derecho están inmersas en un proceso de competencia por jurisdicción entre litigantes docentes y académicos. Ahora veremos, al menos someramente, las consecuencias del triunfo de los segundos en el caso de las facultades norteamericanas ${ }^{26}$.

En Estados Unidos, el proceso de academización de las facultades de derecho está íntimamente relacionado con el éxito, hacia fines del siglo XIX y principios del siglo XX, de las reformas impulsadas por Christopher Columbus Langdell como Decano de Harvard. Langdell entendía la academia universitaria como una profesión en sí misma; si miramos las cosas desde esa perspectiva, un litigante, que es un profesional de la representación de intereses jurídicos ante los tribunales, es en cambio un amateur de la investigación

${ }^{25}$ Comisión Nacional de Acreditación, Acuerdo de Acreditación No 107: Universidad de Atacama, p. 4. Esta resolución rechazó la acreditación de la unidad académica, decisión que fue objeto de una reposición presentada por la institución ante el mismo organismo y donde fue desechada mediante su Acuerdo de Acreditación $N^{o}$ 120. Finalmente, la decisión fue revertida mediante apelación presentada ante el Consejo Nacional de Educación, el que acreditó a la institución por dos años.

${ }^{26}$ Estas líneas, por supuesto, podrían también haber analizado la situación europea. Un elemento, sin embargo, hacen más propicia la realidad norteamericana para su empleo como material para la reflexión local: el hecho de que en Estados Unidos, tal como acá, y a diferencia de la mayoría de los sistemas universitarios europeos, las universidades privadas juegan un rol importante tanto en la producción académica como en la formación profesional. Un importante elemento surge de ahí: la existencia de competencia de mercado entre instituciones.

El modelo norteamericano, como es obvio, contiene importantísimas influencias europeas, particularmente alemanas; y ya ha encontrado recepción en países de otros continentes, como Israel; por lo que calificarle de "norteamericano" es sencillamente una simplificación propia de la construcción de tipos ideales.

Por último, es importante tener presente que los sistemas universitarios norteamericano y europeo tienen definidas ya sea a nivel de legislación (Alemania) o bien a nivel de prácticas unánimemente reconocidas (Estados Unidos) diversos aspectos relacionados con la carrera académica y su estructura interna. Lo que en Chile está tímidamente empezando a delinearse, allá es the law of the land. Véase Muñoz, supra nota 16. 
científica y la enseñanza del derecho. En palabras de James Ames, discípulo de Langdell y su sucesor como Decano de la Facultad de Derecho de Harvard, "no ha de haber duda sobre que el plantel académico deba estar compuesto casi en su totalidad de hombres que dediquen la totalidad de su tiempo a la universidad" 27.

Langdell y Ames fueron los pioneros de la academización actual en las facultades de derecho norteamericanas. Ello se refleja en que, hoy, en Estados Unidos es de sentido común sostener que "los requerimientos profesionales de sus carreras generalmente impide a los docentes a tiempo parcial dedicar una atención substancial a reunirse con los alumnos fuera del salón de clases, así como de participar en el desarrollo del currículum y otros asuntos típicamente conducidos por el cuerpo docente, y de llevar a cabo con seriedad agendas investigativas que contribuyan al desarrollo del derecho" 28 . Es también de sentido común observar que alguien con un nombramiento académico de tiempo completo "puede contribuir al cuerpo del conocimiento con algo que el abogado practicante generalmente no puede: investigación que es rigurosa y sólidamente sustentada" ${ }^{29}$. Es también pacífica, no controversial, la creencia de que los académicos se caracterizan por una mayor independencia de criterio en el análisis del derecho, pues el académico "puede examinar los problemas que involucran al derecho y a las instituciones jurídicas desde un punto de vista ampliamente independiente, careciendo de clientes reales o potenciales que puedan estar mirándolo por sobre el hombro" ${ }^{30}$.

Demos por establecido, en consecuencia, que el proceso de academización que nuestras facultades están viviendo dio sus frutos hace ya bastante tiempo en las facultades de derecho norteamericanas. Esto implica que dichas facultades pueden ser vistas como posibles ejemplos de aquello en lo que nuestras propias facultades se convertirán. Esta posibilidad se ve considerablemente aumentada si tomamos en cuenta la influencia de los académicos que se hayan visto expuestos a la cultura académica norteamericana durante sus estudios de postgrado ${ }^{31}$. En otros países, como Israel, dicha influencia ha sido considerada decisiva en la transformación de la investigación y la enseñanza

${ }^{27}$ Ames, J., "The Vocation of the Law Professor", The American Law Register, Vol. 48, 1900, pp. 129146, p. 137. Véase también Kimball, B., "The Principle, Politics, and Finances of Introducing Academic Merit as the Standard of Hiring for 'the teaching of law as a career,' 1870-1900", Law E Social Inquiry, Vol. 31, 2006, pp. 617-648; y Muñoz, supra nota 5.

${ }^{28}$ Kane, M., "The Requirement of Full-Time Faculty in American Legal Education: Responsibilities and Expectations", Journal of Legal Education, Vol. 51, 2001, pp. 373.

${ }^{29}$ Van Zandt, D., "Discipline-Based Faculty”, Journal of Legal Education, Vol. 53, 2003, p. 333.

${ }^{30}$ Ibídem.

${ }^{31}$ No sería la primera vez que hubiese una influencia explícita de la academia norteamericana en nuestro país. A partir de 1966, la Universidad de Stanford colaboró con un proyecto de reforma de la investigación jurídica nacional que involucró a las universidades de Chile, de Concepción, Católica de Valparaíso, y, en su última etapa, Católica de Chile. Este proyecto de colaboración consistió en un programa de capacitación en métodos de investigación y enseñanza de un total de veinte académicos chilenos a lo largo de un período de tres años, y resultó en la fundación del Instituto de Docencia e Investigaciones Jurídicas en Santiago en 1969. El Instituto desapareció en 1974, cuando se extinguió el financiamiento inicial para este obtenido de la Fundación Ford. Véase Merryman, J., "Law and Development Memoirs I: The Chile Law Program", The American Journal of Comparative Law, Vol. 48, 2000, pp. 481-499. 
jurídicas ${ }^{32}$. ¿Qué prácticas institucionales reflejan la academización de la facultad de derecho norteamericana?

La mejor evidencia de la academización de las facultades norteamericanas se encuentra en el hecho de que el grueso de la planta docente (la faculty) de las escuelas de derecho norteamericanas está integrada por profesores de jornada completa y dedicación absoluta, cuyas posiciones académicas están cubiertas por la garantía de seguridad en el empleo o tenure. El tenure entrega al académico un nombramiento en titularidad caracterizado por el derecho contractual a no ver cesadas sus funciones sin justa causa. En otras palabras, es una especie de derecho o titularidad sobre la posición desempeñada. Los estándares para la regulación del tenure se fundamentan en el Statement of Principles of Academic Freedom and Tenure emitido por la American Association of University Professors en $1940^{33}$. Para el período 2007-2008, por ejemplo, de los 10.673 profesores reportados por las facultades de derecho pertenecientes a la Association of American Law Schools, 7.653 profesores (el $71,7 \%$ ) gozaban de tenure o estaban en posición de adquirirlo ${ }^{34}$. La regulación misma de las facultades, contenida tanto en los Estándares de Acreditación de la American Bar Association como en las directivas autorregulatorias de la Association of American Law Schools, les exige a dichas instituciones "contar con un número suficiente de profesores de jornada completa que asegure la calidad de sus programas educacionales" 35 .

Para los académicos, en consecuencia, la primera y quizás principal meta de desarrollo profesional consiste en alcanzar el tenure. En términos directos el tenure es, entonces, una situación institucional que les entrega estabilidad en el empleo. Pero indirectamente, el tenure es también una señal de prestigio y estatus: socialmente se habla del tenured professor con una cierta reverencia; dentro de la universidad participa de la toma de decisiones colectivas; cuenta con secretarias personales y ayudantes de investigación. Por otro lado, el tenure funciona también como un marcador límite de rendimiento: quien no obtiene tenure dentro del período de cuatro años debe abandonar la institución universitaria y buscar nuevos rumbos profesionales ${ }^{36}$. El tenure se entrega fundamentalmente sobre la base de la producción académica del profesor, expresada en la cantidad y calidad de sus publicaciones y el prestigio de las revistas donde ellas alcanzan difusión. Los candidatos

32 Véase Lahav, P., “American Moment(s): When, How, and Why Did Israeli Law Faculties Come to Resemble Elite U.S. Law”, Theoretical Inquiries in Law, Vol. 10, 2009, pp. 653-697. El autor concluye que, si bien a partir de 1967 "la historia de las facultades de derecho israelíes muestran una creciente dependencia del modelo norteamericano de enseñanza jurídica", "la mayoría de los jóvenes estudiantes que fueron a los Estados Unidos y regresaron a Israel equipados con conocimiento jurídico fueron capaces de absorber dicho conocimiento con una mirada crítica... El resultado han sido instituciones que siguen patrones provenientes de la cultura jurídica norteamericana, pero que mantienen su autonomía y una identidad propia”. Id., pp. 696-697.

${ }^{33}$ Véase Finkin, M.; Post, R., For the Common Good. Principles of American Academic Freedom, New Haven, Yale University Press, 2009.

${ }^{34}$ Association of American Law Schools, Statistical Report on Law Faculty 2007-2008, p. 25.

${ }^{35}$ Kane, M., "The Requirement of Full-Time Faculty in American Legal Education: Responsibilities and Expectations", Journal of Legal Education, Vol. 51, 2001, p. 372.

36 Véase Muñoz, supra nota 16, p. 896. 
saben que su obtención depende de numerosos factores, incluyendo "evaluaciones positivas por parte de los profesores de mayor antigüedad en su área, ubicación de sus artículos en revistas bien evaluadas, numerosas referencias a su trabajo en la literatura pertinente, y la aprobación de colegas de otras áreas" ${ }^{37}$.

¿Qué tan serias son las exigencias de exclusividad de las facultades de derecho para los tenured professors? Al menos, ellas implican que un profesor no puede desempeñarse como tal simultáneamente en más de una universidad. La excepción a esto consiste en el permiso académico (leave of absence), el que faculta al académico a desempeñarse como profesor visitante en otra institución durante un semestre o un año. Esta práctica, relativamente frecuente, le posibilita a los profesores pasar temporadas breves en otras ciudades y otras comunidades académicas. Ahora bien, el tenure, en ocasiones, puede ser suspendido para que el académico se desempeñe en cargos públicos de relevancia, como jefe de alguna agencia reguladora. También ocurre el caso de profesores que buscan acomodar su labor académica con el cargo de juez. Debido a la estructura de la judicatura norteamericana, que no se sustenta en jueces de carrera sino en juristas nombrados tras haberse desempeñado por un cierto tiempo en actividades políticas, litigiosas o académicas, se da el caso de académicos que sean nombrados en cargos judiciales. En estos casos, los académicos suelen mantener un nombramiento de tiempo parcial en la institución universitaria ${ }^{38}$. Por último, la exclusividad sí significa que los académicos no litigan profesionalmente en tribunales. Cabe la posibilidad de que un profesor litigue circunstancialmente un caso de interés público, que ejerza ocasionalmente como consultor, que participe como testigo experto en un juicio o que asesore a un cuerpo legislativo; pero no es común ni bien visto, por ejemplo, que un académico sea socio en una oficina de abogados ${ }^{39}$.

Junto a la tenured faculty se encuentran distintos tipos de adjunct faculty, particularmente los profesores de redacción forense y los profesores de clínicas jurídicas. El origen de estas clínicas jurídicas se remonta a la crítica de los años 30 a la desconexión entre el enfoque teórico de la enseñanza del derecho, que enfatizaba la discusión en clases de los contenidos de sentencias de tribunales de apelación. El pionero de la enseñanza clínica fue el realista jurídico Jerome Frank por medio de su llamado a la creación de "escuelas de abogados" ${ }^{40}$. Sus críticas dieron pie a un amplio movimiento de creación de clínicas jurídicas en las escuelas de derecho, particularmente en las escuelas de "elite". Debido a que no todas las escuelas de derecho cuentan con cursos de redacción o de

${ }^{37}$ Carbado, D., “Tenure”, Journal of Legal Education, Vol. 53, 2003, p. 160.

${ }^{38}$ Como ejemplos destacados podría pensarse en Richard Posner, profesor de la Universidad de Chicago y juez de la United States Court of Appeals for the Seventh Circuit a partir de 1981, y Guido Calabresi, profesor de la Universidad de Yale y juez en la United States Court of Appeals for the Second Circuit a partir de 1994.

${ }^{39}$ Pese a ello, Brian Tamanaha ha observado que en facultades de derecho donde hay poca disciplina y compromiso con la institución es posible que "unos pocos vayan a una oficina donde realicen actividades prácticas en asociación con un estudio de abogados”. Tamanaha, B., Failing Law Schools, Chicago, Chicago University Press, 2012, p. 4.

${ }^{40}$ Frank, J., “A Plea for Lawyer-Schools”, The Yale Law Journal, Vol. 56, 1947, pp. 1303-1344. 
práctica, la existencia al interior de ellas es altamente valorada y constituye un valor distintivo entre distintas escuelas; un elemento que ayuda en la competencia por captar estudiantes talentosos.

Así y todo, el lugar académico de los profesores de dichas asignaturas en la escuela de derecho está delimitado, y consiste en la enseñanza de las asignaturas ya indicadas. En consecuencia, hay una división clara del trabajo: el profesor de planta, un académico con la responsabilidad de producir investigación, entrena a los alumnos en el razonamiento jurídico; mientras que los profesores de la planta adjunta, algunos de los cuales comparten su tiempo entre la docencia y el litigio profesional y otros se dedican solo a la enseñanza de ramos prácticos, están encargados de enseñar destrezas prácticas, ya sea mediante clínicas o mediante cursos de redacción de documentos.

Tras la obtención de tenure, la próxima meta del profesor de derecho es alcanzar una endowed chair, una cátedra nominada financiada mediante el legado de algún donante, y posteriormente alcanzar el estatus de university professor, una especie de supercátedra concedido por algunas universidades y que ocasionalmente lleva el nombre de algún donante particularmente generoso. También es frecuente que un profesor dotado de tenure cambie de una escuela a otra "por razones de sueldo, mejores condiciones laborales, mejor ubicación, mejores colegas y alumnos, y mayor sintonía entre el académico y su institución" ${ }^{41}$. En tales casos, las escuelas le reconocen al profesor el tenure ya adquirido.

El problema que ocurre en algunas facultades de derecho es que, al no estar vinculada a la productividad académica o docente, el tenure permite a los profesores asumir una cómoda posición que le permite hacer el mínimo esfuerzo. Tamanaha ha observado que "prácticamente cualquier facultad de derecho tiene al menos unos pocos profesores que escriben poco", problema frente al cual los decanos de derecho "han mostrado poca disposición a eliminar esta ineficiencia" ${ }^{42}$, particularmente incrementando las cargas docentes de quienes publican menos.

¿Qué mecanismos existen en la actualidad de reclutamiento del personal académico? La forma tradicional de convertirse en profesor de derecho en Estados Unidos es graduarse de una escuela de derecho de prestigio, trabajar en el comité editorial de la revista de mayor prestigio de dicha escuela, publicar un breve artículo o student note, trabajar para un juez federal como asistente o law clerk de uno a tres años, y entonces obtener un trabajo como profesor asistente en una escuela de derecho ${ }^{43}$. Este camino lleva a que el lugar donde un potencial profesor de derecho estudió tiende a prevalecer por sobre sus posteriores méritos académicos, profesionales, o docentes. Así, paradojalmente, "la diversidad racial y de género en las plantas de profesores de derecho ha

${ }^{41}$ Syverud, K., "The Dynamic Market for Law Faculty in the United States”, Journal of Legal Education, Vol. 51, 2001, p. 424. El comentarista nos indica que "el típico profesor se cambiará de escuela al menos una vez".

42 Tamanaha, supra nota 37, p. 45.

${ }^{43}$ Véase Redding, R., “'Where Did You Go to Law School?' Gatekeeping for the Professoriate and Its Implications for Legal Education”, Journal of Legal Education, Vol. 53, 2003, p. 596. 
aumentado drásticamente, pero no ha habido un incremento similar en la diversidad de proveniencia educacional de los nuevos docentes" 44 .

El mercado de entrada a la carrera académica es muy competitivo, pero hay que destacar que está altamente institucionalizado. Cada año, cientos de abogados presentan formularios de registro en el Faculty Appointments Registry de la Association of American Law Schools, la mayoría de los cuales son presentados por vía electrónica ${ }^{45}$. Tras sortear exitosamente la primera etapa de la postulación, el candidato asiste al Faculty Recruitment Conference, una feria laboral anual (conocida informalmente como el meat market) donde se entrevistará brevemente con los comités reclutadores de diversas escuelas. De allí pueden surgir algunas entrevistas (job talks) en las cuales el candidato expondrá, frente a todo el plantel académico de la escuela en cuestión, uno de sus trabajos académicos, usualmente sin publicar todavía (o en estado de work in progress). A esa altura de su vida profesional el candidato debiera ya contar con una o dos publicaciones, por lo que el presentar un trabajo no publicado permite también hacer que la entrevista sea un intercambio académico verdadero, donde eventualmente las indicaciones o sugerencias de los profesores que participan sean tomadas en cuenta en la publicación definitiva.

El carácter profesional de la carrera académica ha creado tendencias que modifican la estructura de reclutamiento ya mencionada. Progresivamente las escuelas de derecho empiezan a buscar candidatos que ya hayan adquirido experiencia en investigación, siguiendo los criterios de contratación de "otras disciplinas académicas, examinando en primer lugar el entrenamiento previo del candidato y sus méritos en materia de publicaciones y calidad de su enseñanza" ${ }^{46}$. Actualmente la tendencia mayoritaria es que los aspirantes a convertirse en profesores postulen a una fellowship académica tras haberse graduado. Dichas fellowships les permiten permanecer uno o dos años más en la escuela de derecho preparando publicaciones que les permitan entrar exitosamente al job market. Otro proceso en marcha es el rol de "la forma de educación jurídica avanzada (J.S.D.)" ${ }^{* 7}$; esto es, la realización de estudios doctorales propiamente tales ${ }^{48}$.

Otra característica importante de la academia norteamericana, como es sabido, es su interdisciplinariedad. Lo interesante es que ella ha desencadenado también cambios paulatinos en el proceso de reclutamiento, resultantes en el incremento en la cantidad de candidatos que han cursado un doctorado en otras áreas del conocimiento. Se ha dicho que debido a ella la escuela de derecho típica "empieza a parecerse más a una universidad en miniatura - sin las ciencias físicas- que a una planta docente en particular dentro de la universidad"; un lugar donde "encontramos filósofos, economistas, críticos literarios,

${ }^{44}$ Ídem., p. 594.

45 Syverud, supra nota 39, p. 423.

${ }^{46}$ Redding, supra nota 41, p. 614.

${ }^{47}$ Redding, supra nota 41, p. 614.

48 Recordemos que en Estados Unidos el título entregado comúnmente por las escuelas de derecho es el de Juris Doctor (J.D.). El Juris Scientiae Doctor (J.S.D.) es, según la jerga académica norteamericana, un research degree; quien lo obtenga debe no solamente haber cursado asignaturas lectivas sino también haber acreditado su capacidad de producir conocimiento mediante la realización de una tesis. Es, en suma, un doctorado. 
académicos de estudios culturales y de estudios femeninos", "historiadores y sociólogos y sicólogos; antropólogos y teólogos” ${ }^{4}$.

Una vez que el académico se ha incorporado a la facultad, ¿cómo se estructura su carga docente? En la actividad del profesor de planta encuentra un lugar protagónico la enseñanza de un número mínimo de cursos semestrales cada año, típicamente tres o cuatro ${ }^{50}$. Normalmente el profesor enseñará algún seminario, alguno de los masivos cursos del currículum de primer año (Constitutional Law, Contracts, Torts, Procedure), y alguno de los cursos más especializados y que no siempre son obligatorios para todos los alumnos (Federal Courts, Criminal Law, Administrative Law, Labor Law, entre otros). Hay que señalar que la estructura de departamentos temáticos existente en Chile y proveniente de la academia europea está ausente de la escuela de derecho norteamericana; no hay departamentos de derecho público, derecho privado, etcétera. Por lo demás, los profesores cruzan frecuentemente las fronteras de lo que para nosotros pertenece a una u otra área del derecho. Esto lleva a que se den casos como los de un profesor enseñando, durante un mismo semestre, filosofía de la acción humana y responsabilidad extracontractual ${ }^{51}$.

Otro elemento central en la vida académica norteamericana es la publicación. Un profesor que no publique constantemente se enfrenta a la presión social de sus pares, fenómeno que es popularmente conocido como publish or perish ${ }^{52}$. La gran paradoja, desde luego, de dicha presión por publicar es que la vida de los académicos (incluyendo su obtención de tenure) depende de las decisiones editoriales de alumnos de segundo año, quienes ofician de directores de prácticamente la totalidad de las revistas de derecho norteamericanas ${ }^{53}$. Entre las críticas que esto despierta hoy se encuentra la afirmación de que los editores estudiantiles se guían en primer lugar por las credenciales académicas de los autores, dejando fuera a los autores cuyas credenciales sean a sus ojos insatisfactorias. Por esto, uno de los procesos de reforma actualmente en curso es la incorporación de profesores con tenure como asesores de las revistas, así como el desencadenamiento de una guerra soterrada (o a veces no tanto) entre revistas student-edited y revistas peer reviewed ${ }^{54}$ por captar ciertos nichos editoriales.

${ }^{49}$ Luban, D., "Legal Scholarship as a Vocation”, Journal of Legal Education, Vol. 51, 2001, p. 168.

${ }^{50}$ Kane, supra nota 28, p. 373.

${ }^{51}$ Estoy pensando en el ejemplo de Jules Coleman, quien el 2008 dictara en la facultad de derecho de Yale al mismo tiempo el curso de Torts y un seminario de filosofía analítica titulado Law and the Reactive Attitudes.

52 Por supuesto, como he observado anteriormente, hay algunos profesores a quienes dicha presión les afecta poco.

${ }^{53}$ Este modelo data de fines del siglo XIX. Con todo, recuerde el lector que las escuelas de derecho en Estados Unidos constituyen una professional school, a la cual se postula tras obtener un bachelor en un college y a menudo tras haber trabajado como paralegal en un estudio jurídico o haber realizado otras labores que permitan incrementar las posibilidades de aceptación en las escuelas de derecho (que no solo exigen un alto puntaje en el Law School Admission Test, sino también la capacidad de mostrar aptitudes profesionales y de liderazgo). El alumno de segundo año de derecho norteamericano, en consecuencia, tiene una formación totalmente distinta a la del alumno de segundo año chileno, quien es básicamente poco más que un egresado de enseñanza media.

${ }^{54}$ Hay que aclarar que ambos tipos de revistas tienen comité editorial; la diferencia es que en un caso son estudiantes y en el otro son profesores (peers). 
Otro elemento central de la práctica académica norteamericana es la existencia de seminarios o workshops organizados regularmente por los profesores. Entre ellos el más importante es el faculty workshop, orientado colectivamente a todos los profesores sin importar su área de especialidad y en el cual se discute semanalmente un paper de elaboración de algún miembro de la comunidad académica. Este seminario "es un elemento ineludible del ambiente de una escuela de derecho hoy, que cumple tanto funciones sociales como intelectuales dentro de la comunidad docente" ${ }^{55}$.

\section{CONCLUSIÓN: EL PROCESO CHILENO EN MARCHA}

Los recientes procesos de ampliación de plantas académicas con académicos graduados en programas doctorales y de dedicación completa parecieran señalar que el modelo tradicional de facultad de derecho está en retirada. Todo esto, cabe destacar, no sugiere que dichas facultades estén olvidando su rol de formadoras de profesionales. Lo que indica es que estaríamos frente a un paulatino triunfo de los académicos en su conflicto con los docentes litigantes sobre la jurisdicción de la enseñanza del derecho.

En ese proceso, las experiencias de otras sociedades que ya han atravesado ese proceso, como la norteamericana, debieran servir como insumo a nuestra discusión local. Algunas de sus instituciones debieran ser revisadas entre nosotros; si bien se hace necesario, como he argumentado anteriormente, la creación de un estatuto que regule y proteja la función académica similar al tenure, también deben estar debidamente reguladas en dicho estatuto la formación y la productividad docente y académica que se le deben exigir al académico. Los mecanismos normativos que sean empleados para alcanzar esta regulación, y que deberán incluir a lo menos estándares legislativamente definidos y criterios reglamentariamente determinados por la autoridad educacional, deberán hacerse cargo de la diversidad del sistema universitario; sin embargo, tal diversidad no puede ser excusa para la consagración de un sistema tan desregulado como el que actualmente existe en la materia.

Adicionalmente, la academización chilena debe estar consciente del peligro de que ella involucre un distanciamiento del ejercicio profesional, como algunas voces críticas sostienen que ha ocurrido en Estados Unidos ${ }^{56}$. En el presente, el mejor argumento de los académicos en su conflicto con los docentes litigantes es que ellos están en mejores condiciones que aquellos de elaborar mecanismos de enseñanza del derecho que potencien la práctica.

Por último, existen prácticas en el academizado medio norteamericano que pueden servir de inspiración para nuestras propias facultades. Esto es así en materia de reclutamiento, donde la existencia de concursos públicos en nuestras facultades de derecho

${ }^{55}$ Lawson, G., "Making Workshops Work”, Journal of Legal Education, Vol. 54, 2004, p. 302.

56 Edwards, H. "The Growing Disjunction between Legal Education and the Legal Profession”, Michigan Law Review, Vol. 91, 1992, pp. 34-70. 
es una práctica que debe ser potenciada, pero que debiera en algún momento alcanzar mayores niveles de publicidad y de estandarización. Esto ocurre también en materia de actividades de reflexión académicas, las cuales en nuestro medio son todavía esporádicas y por lo general no involucran la circulación y discusión de textos académicos.

\section{BIBLIOGRAFÍA}

Аввотт, A., The System of Professions. An Essay on the Division of Expert Labor, Chicago, The University of Chicago Press, 1988.

Altbach, P. et al. (eds.), Paying the Professoriate: A Global Comparison of Compensation and Contracts, New York, Routledge, 2012.

Ames, J., "The Vocation of the Law Professor", The American Law Register, Vol. 48, 1900, pp. $129-146$.

Association of American Law Schools, Statistical Report on Law Faculty 2007-2008.

Carbado, D., "Tenure", Journal of Legal Education, Vol. 53, 2003, pp. 157-173.

Correa, R., "Sistema jurídico chileno", El Mercurio, 20 de enero de 2011, p. A 2.

Durkheim, É., La división social del trabajo, Madrid, Akal, 1987.

Edwards, H., "The Growing Disjunction between Legal Education and the Legal Profession", Michigan Law Review, Vol. 91, 1992, pp. 34-70.

Finkin, M.; Post, R., For the Common Good. Principles of American Academic Freedom, New Haven, Yale University Press, 2009.

Frank, J., “A Plea for Lawyer-Schools”, The Yale Law Journal, Vol. 56, 1947, pp. 1303-1344.

Ingham, J.; Boyle, R., "Generation X in Law School: How These Law Students Are Different From Those Who Teach Them”, Journal of Legal Education, Vol. 56, 2006, pp. 281-295.

Kane, M., "The Requirement of Full-Time Faculty in American Legal Education: Responsibilities and Expectations", Journal of Legal Education, Vol. 51, 2001, pp. 372-374.

KANT, I., El conflicto de las facultades, Madrid, Alianza Editorial, 2003.

Krieger, S., "The Development of Legal Reasoning Skills in Law Students: An Empirical Study", Journal of Legal Education, Vol. 56, 2006, pp. 332-355.

Kimball, B., "The Principle, Politics, and Finances of Introducing Academic Merit as the Standard of Hiring for 'the teaching of law as a career,' 1870-1900”, Law E Social Inquiry, Vol. 31, 2006, pp. 617-648.

Lahav, P., "American Moment(s): When, How, and Why Did Israeli Law Faculties Come to Resemble Elite U.S. Law”, Theoretical Inquiries in Law, Vol. 10, 2009, pp. 653-697.

Lawson, G., "Making Workshops Work". Journal of Legal Education, Vol. 54, 2004, pp. 302-312. LubAN, D. "Legal Scholarship as a Vocation", Journal of Legal Education, Vol. 51, 2001, pp. 167-174.

Merryman, J., "Law and Development Memoirs I: The Chile Law Program", The American Journal of Comparative Law, Vol. 48, 2000, pp. 481-499.

MuÑOZ, F., "Langdell's and Holmes's influence on the institutional and discursive conditions of American legal scholarship”, Revista Chilena de Derecho, Vol. 38, 2011, pp. 217-237.

MuÑoz, F., "La necesidad de regular la docencia universitaria en Chile: una propuesta de lege ferenda", Revista Chilena de Derecho, Vol. 39, 2012, pp. 891-907.

RedDing, R., “'Where Did You Go to Law School?' Gatekeeping for the Professoriate and Its Implications for Legal Education”, Journal of Legal Education, Vol. 53, 2003, pp. 594-614.

Pargendler, M., "The Rise and Decline of Legal Families", American Journal of Comparative Law, Vol. 60, 2012, pp. 1043-1074. 
Rodríguez, P., "Misión profesional de la universidad”, El Mercurio, sábado 23 de mayo de 2009, A 2.

Rodríguez, P., "Sistema jurídico chileno", El Mercurio, miércoles 19 de enero de 2011, A 2.

Simmel, G., Sobre la individualidad y las formas sociales, Buenos Aires, Universidad Nacional de Quilmes, 2002.

Syverud, K., "The Dynamic Market for Law Faculty in the United States", Journal of Legal Education, Vol. 51, 2001, pp. 423-426.

Tamanaha, B., Failing Law Schools, Chicago, Chicago University Press, 2012.

VAn ZandT, D., "Discipline-Based Faculty”, Journal of Legal Education, Vol. 53, 2003, pp. 332-339.

Weber, M., Economía y sociedad. Esbozo de sociología comprensiva, México, Fondo de Cultura Económica, 1964.

Weber, M., El político y el científico, Madrid, Alianza Editorial, 2005.

Zweigert, K., y Kötz, H., Introduction to Comparative Law, Oxford, Oxford University Press, 1998. 